\title{
DETERMINACIÓN DE GÉNEROS BACTERIANOS EN EL MAR DE LA PLAYA CANTOLAO - LA PUNTA - CALLAO
}

\author{
Justo $S$, \\ Churasacari $T$, \\ Saldaña $C$, \\ Cajachagua $C$, \\ Pabón L, \\ Gónzales $M$, \\ Santiago $D$, \\ Guerra A.
}

\section{RESUMEN}

Los océanos albergan una gran diversidad biológica, hasta el momento relativamente poco explorada por el humano. Con el objetivo de ampliar los conocimientos en la biodiversidad bacteriana del mar peruano, se realizaron muestreos aleatorios en el mar de Playa Cantolao-La Punta-Callao (12 $\left.04^{\prime} 06^{\prime \prime} \mathrm{S} 77^{\circ} 10^{\prime} 10^{\prime \prime} \mathrm{O}\right)$, en total se tomaron 5 muestras en frascos estériles a una profundidad de $3 \mathrm{~m}$ utilizando una vara de madera acondicionada a una jeringa de $20 \mathrm{ml}$. Para el aislamiento se utilizó el método de dispersión en placa en Agar Marino, se trabajó con diluciones sucesivas para obtener colonias aisladas. Para la identificación se realizaron pruebas bioquímicas que incluían determinación de citocromo oxidasa, motilidad, producción de $\mathrm{H}_{2} \mathrm{~S}$, producción de indol, metabolismo de glucosa, producción de pigmento, luminiscencia, catalasa y desarrollo en anaerobiosis; además de las coloraciones Gram y de espora. Los esquemas propuestos por Das S et al. (2007), Oliver J (1982) y el Bergey's Manual 2da y 7ma edición fueron utilizados para la determinación de géneros. Se logró el aislamiento de 20 cepas bacterianas. Los géneros encontrados fueron: Moraxella, Bacillus, Acinetobacter, Vibrio, Aeromonas, Photobacterium, Alteromonas, Alcaligenes, Streptococcus y Chromobacterium.

Palabras Claves: Bacterias Marinas, Esquemas de Das S et al (2007) y Oliver J (1982), Pruebas bioquímicas.

Laboratorio de Microbiología. Facultad de Ciencias Biológicas. Universidad Ricardo Palma.sanjusare_712@hotmail.com 


\begin{abstract}
The oceans are home to a rich biodiversity, until now relatively little explored by humans. In order to broaden the knowledge on bacterial biodiversity of the Peruvian sea, random samples were taken at sea of Playa Cantolao - La Punta - Callao (12 $04^{\prime} 06^{\prime \prime}$ $\mathrm{S} 77^{\circ} 10^{\prime} 10^{\prime \prime} \mathrm{O}$ ), in total were taken 5 samples in sterile bottle to a depth of $3 \mathrm{~m}$ using a wooden stick fitted to a $20 \mathrm{ml}$ syringe. To isolate we used the dispersion method in plates with Marine Agar, we worked with successive dilutions for the obtaining of isolated colonies. Biochemical tests for identifying including cytochrome oxidase determination, motility, $\mathrm{H}_{2} \mathrm{~S}$ production, indol production, glucose metabolism, pigment production, luminescence, catalase and anaerobically development. The schemes proposed by Das S et al. (2007), Oliver J (1982) and the $2^{\text {nd }}$ and $7^{\text {th }}$ edition of the Bergey's Manual were used to determine the genera. We achieved the isolation of 20 bacterial strains. The genera found were: Moraxella, Bacillus, Acinetobacter, Vibrio, Aeromonas, Photobacterium, Alteromonas, Alcaligenes, Streptococcus y Chromobacterium.
\end{abstract}

Keys words: Marine Bacteria, Schemes of Das S et al (2007) and Oliver J (1982), Biochemical Tests.

\title{
INTRODUCCIÓN
}

Los océanos comprenden aproximadamente el $70 \%$ de la superficie terrestre; la diversidad de sus ambientes permite que se desarrolle gran cantidad de formas de vida; además tienen importancia como regulador de la temperatura global y como sumidero de carbono.

De esta primera idea se desprenden dos principales: la diversidad de especies y la importancia ecológica de los ecosistemas marinos.

En primer lugar, la diversidad biológica marina comprende todo tipo de organismos que habiten en los mares, un grupo importante con poca consideración en nuestro país son las bacterias; estudios recientes en otras partes del mundo han hallado bacterias marinas con propiedades que van desde 
la producción de pigmentos utilizables (Yada S. et al. 2007) hasta bacterias que purifican nanopartículas de oro a partir de otros compuestos (Shama, N. et al. 2012), además de bacterias ampliamente estudiadas como Marinobacter hydrocarbonoclasticus capaz de producir enzimas que degradan hidrocarburos aromáticos, Vibrio fischeri que tiene en su genoma el operon LUX (biolumniscencia) o Pseudomonas pseudoalcaligenes productora de enzima que degrada el cianuro.

En segundo lugar, el bacterioplancton tiene un papel significativo como principal contribuyente en los procesos biogeoquímicos de los ambientes marinos, y representa un componente fundamental en las redes alimentarias. En los últimos años, el concepto de red trófica clásica ha cambiado a un modelo alternativo más complejo denominado "bucle microbiano", basado en la capacidad de las bacterias heterótrofas de reciclar la materia orgánica disuelta y de hacerla circular mediante diversas interacciones ecológicas a través de los otros componentes del plancton y, en forma indirecta, también a través de los niveles tróficos superiores del ecosistema marino (Schauer, M. et al. 2011). En los océanos, los microorganismos son los responsables del $98 \%$ de la producción primaria e intervienen en todos los ciclos de la materia, con un papel fundamental, a su vez, en el destino de los contaminantes en zonas altamente antropizadas. Además los organismos heterótrofos como las bacterias consumen entre el 18\% y $77 \%$ de carbono orgánico disuelto en el mar (Azam, F. et al. 1983).

Por lo tanto el estudio de las bacterias en los ambientes marinos de nuestro país nos permitirá aumentar nuestros conocimientos en la ecología y buscar nuevos recursos aprovechables. El presente estudio tiene como objetivo identificar los géneros bacterianos presentes en el mar de Playa CantolaoCallao como base para futuros estudios ecológicos y biotecnológicos.

\section{MATERIALES Y MÉTODOS}

Recolección de muestras:

Se realizaron muestreos aleatorios en el mar de la playa Cantolao-La Punta-Callao(12 $\left.04^{\prime} 06^{\prime \prime} \mathrm{S} 77^{\circ} 10^{\prime} 10^{\prime \prime} \mathrm{O}\right)$. Se tomaron 5 muestras, a una profundidad de aproximadamente 3 metros utilizando una vara de madera acondicionada con jeringas estériles de $20 \mathrm{ml}$. Además se colectaron 3 litros de agua de mar para la preparación de los medios de cultivo. Las 
muestras de agua fueron transportadas al Laboratorio Microbiología de la Universidad Ricardo Palma en un cooler refrigerado a $4^{\circ} \mathrm{C}$.

Aislamiento de bacterias:

Se utilizó el método de dispersión en placa con medio marino (fig. 1). Para esto se tomó $1 \mathrm{ml}$ de la muestra y se colocó en un tubo con $9 \mathrm{ml}$ de agua de mar esterilizada y filtrada obteniendo la dilución $10^{-1}$, se repitió este procedimiento obteniendo 3 diluciones (hasta $10^{-3}$ ). Las placas fueron incubadas a $\mathrm{T}^{\mathrm{o}}$ ambiente $\left(20-25^{\circ} \mathrm{C}\right)$ durante $48 \mathrm{~h}$. Al cabo de este tiempo se seleccionaron las colonias aisladas y fueron reaisladas en tubos con Agar Marino. Estas fueron incubadas nuevamente a $\mathrm{T}^{\mathrm{o}}$ ambiente hasta observar crecimiento visible y luego fueron almacenadas a $4^{\circ} \mathrm{C}$ hasta el momento de la caracterización bioquímica.

Determinación Bioquímica:

Se realizaron las siguientes pruebas bioquímicas: coloración gram, citocromo oxidasa, motilidad, producción de $\mathrm{H}_{2} \mathrm{~S}$, producción de indol, metabolismo de glucosa, producción de pigmento, luminiscencia, catalasa, desarrollo en anaerobiosis, coloración de esporas. (fig.2)

Los esquemas propuestos por Das, S. et al. (2007), Oliver J (1982) y el Bergey's Manual 2da y $7 \mathrm{ma}$ edición fueron utilizados para la determinación de géneros.

\section{RESULTADOS}

En total se obtuvieron 20 cepas, de las cuales 17 lograron ser identificadas hasta la categoría de género; 3 no tuvieron crecimiento suficiente para su identificación.

Se encontraron 10 géneros. De los cuales, 8 fueron bacilos gramnegativos y sólo 2 grampositivos, un bacilo y un coco (tabla1).

\section{CONCLUSIONES}

Se determinó la presencia de 10 géneros bacterianos, estos fueron: Moraxella (23,5\%), Bacillus (5,88\%), Acinetobacter (5,88\%), Vibrio (11,76\%), Aeromonas (11,76\%), Photobacterium (5,88\%), 
Alteromonas (11,76\%), Alcaligenes (5,88\%), Streptococcus (5,88\%) y Chromobacterium (11,76\%).

\section{DISCUSIONES}

Pellón et al. (2001) en su trabajó con muestras de 2 moluscos bivalvos cultivados en Pucusana y el Callao: Argopecten purpuratus "concha de abanico" y Crassostrea gigas "ostra", logran identificar 6 géneros bacterianos: Vibrio (40\%), Aeromonas (15\%), Flavobacterium (10\%), Pseudomonas (5\%), Moraxella (5\%), Flexibacter (5\%) y no identificados (20\%), León et al. (2010) por su parte en su trabajó con muestras de 5 invertebrados intermareales recolectados en la Bahía de Ancón: Phymactis clematis "anémona", Tetrapygus niger "erizo de mar", Heliaster helianthus "estrella de mar", peje sapo y Tegula atra "caracol" logra identificar 4 géneros: Vibrio (50\%), Pseudomonas $(10 \%)$, Flavobacterium (20\%) y Actinomycete (20\%), el presente trabajo reporta la presencia en el ambiente marino de 10 géneros bacterianos entre ellos Aeromonas, Moraxella y Vibrio sin embargo no se encontró cepas de Flavobacterium, Flexibacter ni Actynomicetes lo que sugiere que estos géneros podrián estar más relacionados a organismos que géneros como Bacillus, Photobacterium y Alteromonas que no fueron encontrados en los trabajos mencionados anteriormente pero sí en este. Pellón et al. (2001) y León et al. (2010) determinan que la cepa más abundante es la correspondiente a Vibrio; sin embargo, en este trabajo la cepa más abundante fue la de Moraxella, esto puede ser a causa de que hay mayor asociación de Vibrio con invertebrados que el género Moraxella. Además en un estudio realizado en Argentina, en la zona costanera de Comodoro se reporta en muestras de sedimentos y agua marina 6 de los géneros hallados en este trabajo (Acinetobacter, Aeromonas, Vibrio, Moraxella, Bacillus, Photobacterium), adicionalmente determinan que la mayor cantidad de cepas halladas fueron grampositivas a diferencia del presente trabajo que encuentra que el $80 \%$ de los géneros y el $90 \%$ de las cepas aisladas fueron gramnegativas, esto sugiere y afirma que las grampositivas están mayormente asociados a los suelos y que las gramnegativas más bien habitan asociadas a la columna de agua. 


\section{REFERENCIAS BIBLIOGRÁFICAS}

1. Azam, F.; Fenchel, T.; Field, J.; Gray, J.; Meyer-Reil, L. y Thingstad, F. 1983. The ecological role of water-column microbes in the sea. Marine Ecology Progress Series, 10: 257-263.

2. Das, S.; Lyla, P. y Ajmal, S. 2007. A simple scheme for the identification of marine heterotrophic bacteria. An international journal of marine sciences Thalassas, 23(2): 17-21.

3. Kaysner, C. y De Paola, A. 2004. BacteriologicalAnalytical Manual. FDA (internet). (citado el 09 de Febrero del 2014). Disponible en: http://www.fda.gov/Food/FoodScienceResearch/LaboratoryMethods/ucm070830.htm

4. Oliver, J. 1982. Taxonomic scheme for the identification of marine bacteria. Deep Sea Research, 29(6A): 795-798.

5. Pucci, G.; Acuña, A.; Llanes, M.; Tiedemann, M. y Pucci, O. H.. 2009. Identificación de bacterias marinas cultivables de la ciudad costera Comodoro Rivadavia, Argentina. Revista de biología marina y oceanografia, 44(1), 49-58.

6. Schauer, M.; Balagué, V.; Pedros-Alio, C. y Massana, R. 2003. Seasonal changes in the taxonomic composition of bacterioplankton in a coastal oligotrophic system. Aquatic Microbial Ecology, 31: 163-74.

7. Shama, N.; Pinnaka, A.; Raje, M.; Fnu, A.; Sharkar, M. y Roy, A. 2012. Exploitation of marine bacteria for production of gold nanoparticles. Microbial cell factories, 11: 86-92.

8. Shikongo, M.; Chimwamurombe, P. y Venter, S. 2012. Identification of putative Vibrio species isolated from processed marine fish using thiosulphate-citrate-bile-sucrose (TCBS) agar. British biotechnology journal, 2(4): 229-246.

9. Yada, S.; Wang, Y.; Zou, Y.; Nagasaki, K.; Hosokawa, K.; Osaka, I.; Arakawa, R. y Enomoto, K. 2008. Isolation and characterization 
of two groups of novel marine bacteria producing violcain. Marine biotechnology, 10: 128-132.

10. Pellón, F.; Orozco, R. y León, J. 2001. Bacterias marinas con capacidad antimicrobiana aisladas de moluscos bivalvos en cultivos. Rev. Per. Biol. Vol. 8. № 2 .

11. León, J.; Liza, L.; Soto, I.; Torres M. y Orosco, A. 2010.Bacterias marinas productoras de compuestos antibacterianos aisladas a partir de invertebrados intermareales. RevPeruMedExp Salud Pública, 27(2): $215-21$.

12. Ruíz, C. 2013. Respuesta antagónica de microorganismos aislados de los cultivos de microalgas en el laboratorio de cultivos marinos. DELOS Desarrollo Local Sostenible Grupo Eumed.net / Universidad de Málaga y Red Académica Iberoamericana Local Global. Vol 6. $\mathrm{N}^{\mathrm{o}} 18$.

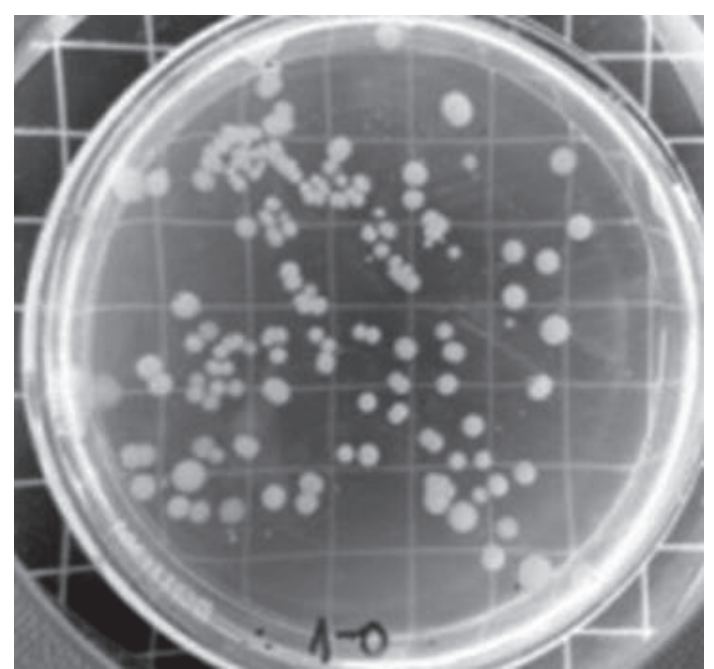

Fig. 1. Aislamiento por diluciones sucesivas en medio Marino. 


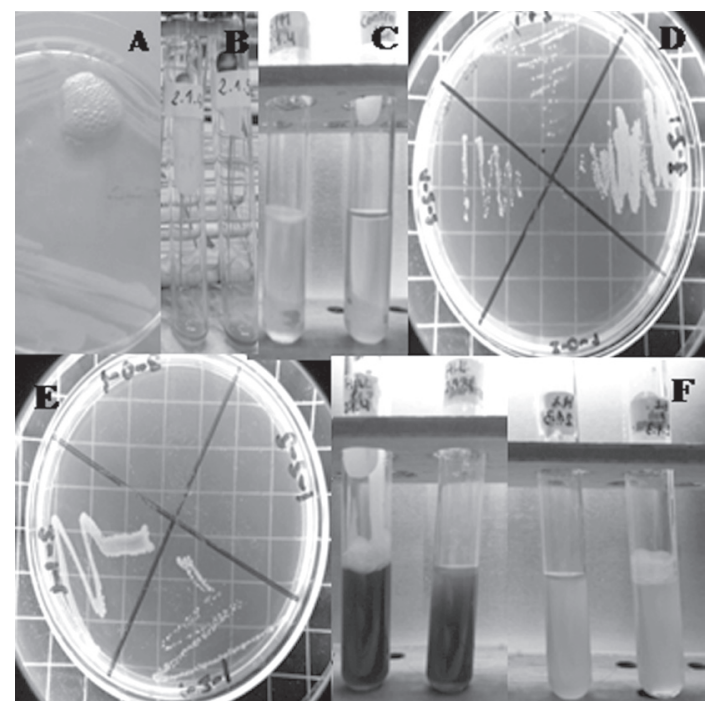

Fig. 2. A; Prueba de catalasa, B; Citocromo oxidasa, C; SIM, D; Agar $\mathrm{P}$ (pigmentación), E; Agar Marino (Anaerobiosis), F; HughLeifson (metabolismo de glucosa).

TABLA 1. Resultados de las pruebas bioquímicas de las 17 cepas analizadas

\begin{tabular}{|c|c|c|c|c|c|c|c|c|c|c|c|c|c|}
\hline & CAT. & OXID. & INDOL & MOT. & $\mathrm{H}_{2} \mathrm{~S}$ & PIG. & LUMINISC. & GRAM & ANAEROB. & ESPORA & & & ESPECIE \\
\hline 1.3 & & & & & & & & & & & $A b$ & Cer & \\
\hline 1.3 & - & + & & - & - & - & - & B- & + & NP & + & - & Moraxella \\
\hline 1.4 & + & + & - & + & - & - & - & B- & + & NP & + & - & Alteromonas \\
\hline 1.5 & + & $\mathbf{N}$ & - & - & - & - & - & $\mathrm{B}+$ & + & Central & - & - & Bacillus \\
\hline 1.6 & + & - & - & - & - & - & - & B- & + & NP & + & + & Acinetobacter \\
\hline 1.7 & + & + & - & + & - & - & - & B- & + & NP & + & + & Vibrio \\
\hline 1.8 & + & + & + & - & - & - & - & B- & + & NP & + & + & Photobacterium \\
\hline 1.9 & + & + & - & - & - & - & - & B- & + & NP & + & + & Moraxella \\
\hline 1.10 & - & + & - & - & - & - & - & B- & + & NP & + & - & Moraxella \\
\hline 2.1 & + & - & - & + & $\cdot$ & - & - & B- & + & NP & + & - & Alcaligenes \\
\hline 2.2 & - & + & - & - & - & - & - & C+ & - & NP & - & - & Streptococcos \\
\hline 2.3 & - & + & + & + & - & - & - & B- & - & NP & + & + & Aeromonas \\
\hline 2.4 & + & + & + & + & - & - & - & B- & + & NP & + & + & Vibrio \\
\hline 2.5 & + & + & - & + & - & - & - & B- & + & NP & + & - & Chromobacterium \\
\hline 2.6 & - & + & + & + & - & - & - & B- & - & NP & + & + & Aeromonas \\
\hline 2.7 & + & + & $\cdot$ & $\cdot$ & $\cdot$ & $\cdot$ & - & B- & + & NP & + & $\cdot$ & Moraxella \\
\hline 2.8 & + & + & - & + & $\cdot$ & - & - & B- & + & NP & + & - & Alteromonas \\
\hline 2.9 & + & + & - & + & - & - & - & B- & + & NP & + & - & Chromobacterium \\
\hline
\end{tabular}

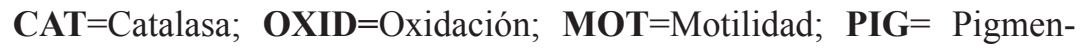
tación; ANAEROB=Anaerobiosis; H.F = HughLeifson; $\mathbf{A b}=$ Aerobio, $\mathbf{C e r}=$ Anaerobio; $\mathbf{N P}=$. No hay presencia de espora; $\mathbf{N}=$ No se realizó la prueba, 